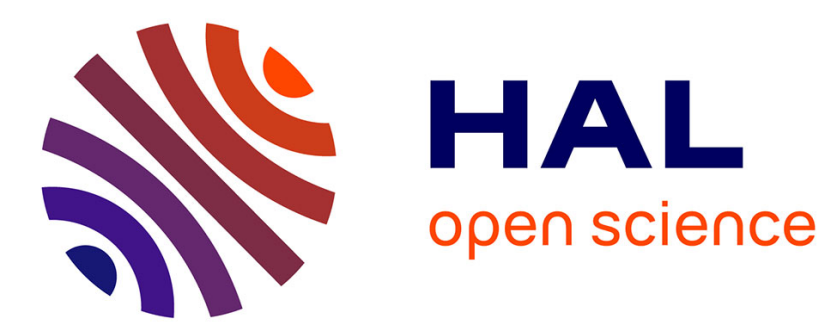

\title{
Structural modal interaction of a four-degree-of-freedom bladed disk and casing model
}

\author{
Mathias Legrand, Christophe Pierre, Bernard Peseux
}

\section{To cite this version:}

Mathias Legrand, Christophe Pierre, Bernard Peseux. Structural modal interaction of a four-degreeof-freedom bladed disk and casing model. Journal of Computational and Nonlinear Dynamics, 2010, 5 (4), pp.13-41. 10.1115/1.4001903 . hal-00522524

\section{HAL Id: hal-00522524 \\ https://hal.science/hal-00522524}

Submitted on 30 Sep 2010

HAL is a multi-disciplinary open access archive for the deposit and dissemination of scientific research documents, whether they are published or not. The documents may come from teaching and research institutions in France or abroad, or from public or private research centers.
L'archive ouverte pluridisciplinaire HAL, est destinée au dépôt et à la diffusion de documents scientifiques de niveau recherche, publiés ou non, émanant des établissements d'enseignement et de recherche français ou étrangers, des laboratoires publics ou privés. 


\title{
Structural modal interaction of a four degree of freedom bladed disk and casing model
}

\author{
Mathias Legrand \\ Institut de Recherche en Génie civil et Mécanique (GeM), UMR CNRS 6183, École Centrale de \\ Nantes, BP 92101, 44321 Nantes cedex 3, France
}

\section{Christophe Pierre}

Structural Dynamics and Vibration Laboratory, Department of Mechanical Engineering, McGill University, 817 Sherbrooke St West, Montreal, Quebec H3A 2K6, Canada

\section{Bernard Peseux}

Institut de Recherche en Génie civil et Mécanique (GeM), UMR CNRS 6183, École Centrale de Nantes, BP 92101, 44321 Nantes cedex 3, France

\begin{abstract}
Consideration is given to a very specific interaction phenomenon that may occur in turbomachines due to radial rub between a bladed disk and surrounding casing. These two structures, featuring rotational periodicity and axi-symmetry respectively, share the same type of eigenshapes, also termed $n_{d}$-nodal diameter traveling waves. Higher efficiency requirements leading to reduced clearance between blade-tips and casing together with the rotation of the blade disk increase the possibility of interaction between these traveling waves through direct contact. By definition, large amplitudes as well as structural failure may be expected. A very simple two-dimensional model of outer casing and bladed disk is introduced in order to predict the occurrence of such phenomenon in terms of rotational velocity. In order to consider traveling wave motions, each structure is represented by its two $n_{d}$-nodal diameter standing modes. Equations of motion are solved first using an explicit time integration scheme in conjunction with the Lagrange multiplier method which accounts for the contact constraints, and then by the Harmonic Balance Method. While both methods yield identical results that exhibit two distinct zones of completely different behaviors of the system, HBM is much less computationally expensive.
\end{abstract}

Keywords: modal interaction wave speed coincidence explicit time marching procedure harmonic balance method

\section{Introduction}

There is a broad variety of mechanisms leading to rotor/stator contact induced vibration problems in turbo-machinery including rotor imbalances due to design uncertainties, blade-offs or bird strikes, thermal gradients in outer casings, aerodynamic excitations of vibration modes, sudden rotor fatigue cracks. The latter may be classified according to the interaction locations, either rotating shafts touching retainer bearings or blade-tips contacting the stationary outer casing. Depending on the complexity of the chosen modeling, a broad class of phenomena can be observed.

In the past, attention was mostly paid to the vibrations of rotating shafts supported by journal bearings where different levels of nonlinearity were considered: oil-film pressure field implicating nonlinear hydrodynamic equations, direct rub and friction forces, viscous damping forces $[1,2,3]$. Models generally involved an in-plane Jeffcott rotor housed within the clearance of a simplistic stator where a unique contact location was accounted for along the circumferential direction. The reader may want to consult the rich literature related to the resulting nonlinear and chaotic behaviors as different as dry whip, oil whip or whirling motion [4, 5], to name a few.

Recently, problems more specific to aircraft engines have arisen [6]. Due to the constant need of higher efficiency, undesired phenomena involving direct contact may be detected. Indeed, this 
efficiency, simply defined as the ratio of energy output to energy input, strongly depends on the tip clearance between the rotating and stationary parts: the wider the clearance, the less efficient the machine. A reduced clearance between the two components significantly increases the occurrence of contact that is no longer limited to only one simultaneous location. Consequently, it becomes apparent that fully flexible bladed disk and outer casing models are required for a realistic description of the interaction for which unexpected instabilities are identified [7, 8]. Understanding the physical mechanisms that cause them and the circumstances under which they occur is of utmost importance for engineers and designers. They can be classified into two categories [9]:

- transitional phenomena (no more than several milliseconds) that can generate very high stresses in the structures. This is what happens after a blade-off, for instance, where the mechanical components are subjected to very high deceleration rates due to the stop of the engine.

- long-lived phenomena (several rounds of the rotor) characterized by intermittent soft contacts that may induce very high stress levels due to a forced excitation of the blade modeshapes resulting in the fragmentation of the tip of the latter or due to a coupling between the nodal diameter vibration modes of the casing and their counterparts of the bladed disk.

The primary goal of the present paper is to give a better understanding of the second type of interaction between the stator and the rotor, called "modal interaction" in the sequel. To the best of our knowledge, only one particularly interesting and relevant dissertation by Schmiechen [10] and a paper [11] deal with this traveling wave speed coincidence.

To this end, a simple numerical tool, that relies on two elastic structures, has been developed. Each structure is represented in terms of its two $n_{d}$-nodal diameter vibration modes which allow traveling wave motion. The kinetic energy of the rotor is transformed into vibratory energy thanks to direct contact between the two structures and may result in a dangerous situation of interaction. In this numerical tool, the equations of motion are solved using two different approaches:

- a time-stepping method based on the explicit central differences scheme, relevant for dynamical systems with contact constraints [12]. This method involves a prediction-correction procedure in conjunction with the calculation of Lagrange multipliers to account for the contact efforts.

- a frequency domain approach, the Harmonic Balance Method, that requires prior determination of the retained harmonic terms and involves elaborate algebraic formulation but which is one of the most commonly used approaches for analyzing strong nonlinear dynamic systems [13].

The paper concludes on a series of evolutions of the proposed model for future investigations.

\section{Mathematical background}

The concept of $n_{d}$-nodal diameter traveling wave is briefly summarized in a simple fashion. Rotational periodicity and axi-symmetry, both characteristic of bladed disks and outer casings in turbomachines, give rise to mode pairs with identical natural frequencies [14]. Both respective mode shapes are similar and rotated around the axis of symmetry by $\pi /\left(2 n_{d}\right)$ where $n_{d}$ is the number of nodal diameters of the modes. Due to the orthogonality property of normal modes, it is possible to combine them into forward and backward traveling waves. Let $\theta$ be an angular position of a typical material point and $\gamma$, an amplitude of displacement of this material point. These two traveling waves can be expressed as:

$$
\begin{aligned}
& \gamma_{f}(t, \theta)=\gamma_{0} \cos \left(\omega t-n_{d}\left(\theta-\theta_{0}\right)\right) \\
& \gamma_{b}(t, \theta)=\gamma_{0} \cos \left(\omega t+n_{d}\left(\theta-\theta_{0}\right)\right)
\end{aligned}
$$


where $\omega$ is the angular frequency and $\theta_{0}$, an undetermined angular position. The scalar $\gamma_{0}$ stands for the amplitude of $\gamma_{f}$ and $\gamma_{b}$ which are, respectively, the forward and backward traveling waves. In a body-fixed reference frame, the propagation velocity of these two waves is $\pm \omega / n_{d}$ according to their direction. For a structure rotating at a uniform angular velocity $\Omega$ relative to a stationary frame, the propagation velocity of these two waves in a stationary frame becomes $\Omega+\omega / n_{d}$ for the co-rotating wave and $\Omega-\omega / n_{d}$ for the counter-rotating wave, selecting the counter-clockwise direction as positive. The primary goal of this research project is to detect a structural interaction due to direct contact between these types of waves, one possibly traveling in the casing and the second one in the bladed disk.

\section{Structural models}

\subsection{Bladed disk}

In this study, the model of the bladed disk is as simple as possible. Each blade of the tuned assembly is represented by a rigid body linked to the disk by a linear spring $k_{b}$, and to its neighbors by a linear spring $k_{b b}$, as depicted in Fig. 1. For the sake of simplicity in the contact detection explained later, it is assumed that the blades modeled as such vibrate around the axis of rotation of the engine or equivalently that the radius of the disk collapses to zero. The stiffening due to centrifugal forces is neglected as well as gyroscopic effects. Hence, the dynamic equation associated with the $i$-th blade

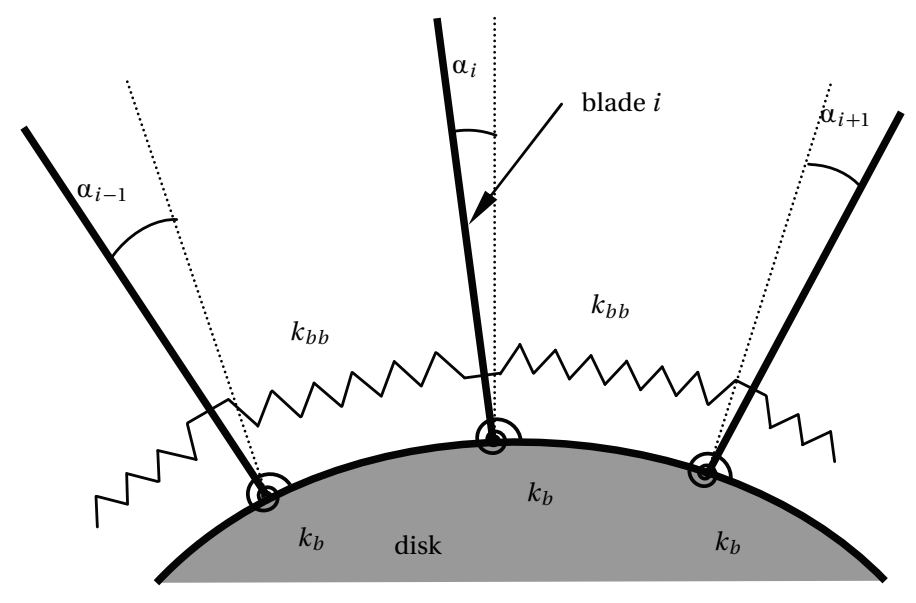

Figure 1: Schematic of the bladed disk

(or rigid body) is:

$$
m_{b} \ddot{\alpha}_{i}+\left(k_{b}+2 k_{b b}\right) \alpha_{i}-k_{b b}\left(\alpha_{i+1}+\alpha_{i-1}\right)=\mathrm{F}_{i}
$$

where $\alpha_{i}$ represents the vibration angle of the $i$-th blade and $\mathrm{F}_{i}$, the respective external forcing acting on that blade. This equation becomes, when generalized to the entire structure:

$$
\mathbf{M}_{b d} \ddot{\boldsymbol{\alpha}}+\mathbf{K}_{b d} \boldsymbol{\alpha}=\mathbf{F}_{b d}
$$

The mass matrix contains on its diagonal the moment of inertia of a rigid beam about one of its ends, $m_{b}$ such that $\mathbf{M}_{b d}=\operatorname{diag}_{\mathrm{N}}\left(m_{b}\right)$. Noting $k_{1}=k_{b}+2 k_{b b}$ and $k_{2}=-k_{b b}$, the circulant stiffness matrix is $\mathbf{K}_{b d}=\operatorname{circ}_{\mathrm{N}}\left(k_{1}, k_{2}, 0, \ldots, 0, k_{2}\right)$. The two resulting $n_{d}$-nodal diameter modes $\mathbf{p}_{\mathbf{c}}$ and $\mathbf{p}_{\mathbf{s}}$, illustrated in Fig. 2 for $n_{d}=3$, are associated with their respective contributions $\alpha_{c}$ and $\alpha_{s}$ so that the final displacement field of the structure is:

$$
\left(\begin{array}{c}
\alpha_{1} \\
\vdots \\
\alpha_{N}
\end{array}\right)=\left[\begin{array}{ll}
\mathbf{p}_{\mathbf{c}} & \mathbf{p}_{\mathrm{s}}
\end{array}\right]\left(\begin{array}{c}
\alpha_{c} \\
\alpha_{s}
\end{array}\right)
$$


Accordingly, a $n_{d}$-nodal diameter traveling wave will propagate in the bladed disk when considering the following time functions $\alpha_{c}(t)=\cos (\omega t)$ and $\alpha_{s}(t)=\sin (\omega t)$, for instance.

\subsection{Casing}

The casing is directly discretized onto its modal coordinates by only keeping its two $n_{d}$-nodal diameter modes as shown in Fig. 2. Their respective contributions are denoted $u_{c}$ and $u_{s}$. Any material point belonging to the casing, whose position is parameterized by $\theta$, is then subjected to the following displacement field:

$$
u(t, \theta)=u_{c}(t) \cos \left(n_{d}\left(\theta-\theta_{0}\right)\right)+u_{s}(t) \sin \left(n_{d}\left(\theta-\theta_{0}\right)\right)
$$

As already explained for the bladed disk, a traveling wave will propagate in the casing if, for instance $u_{c}(t)=\cos (\omega t)$ and $u_{s}(t)=\sin (\omega t)$. Noting $\mathbf{u}=\left[u_{c} u_{s}\right]^{\mathrm{T}}$, the two dynamic equations of the casing are recast in the following contracted form:

$$
\mathbf{M}_{c} \ddot{\mathbf{u}}+\mathbf{K}_{c} \mathbf{u}=\mathbf{F}_{c}
$$

where $\mathbf{F}_{c}$ is an external load applied to the casing. The mass matrix contains the modal mass of the two considered modes on its diagonal, $\mathbf{M}_{c}=\operatorname{diag}_{2}\left(m_{c}\right)$ and the respective modal stiffness matrix is written as $\mathbf{K}_{c}=\operatorname{diag}_{2}\left(k_{c}\right)$.

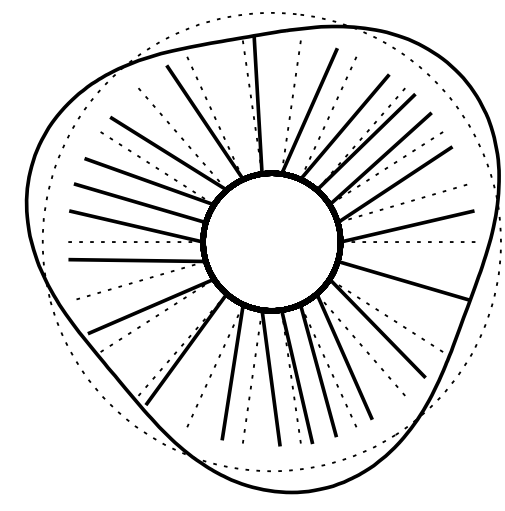

(a) cosine modes $u_{c}$ and $\alpha_{c}$

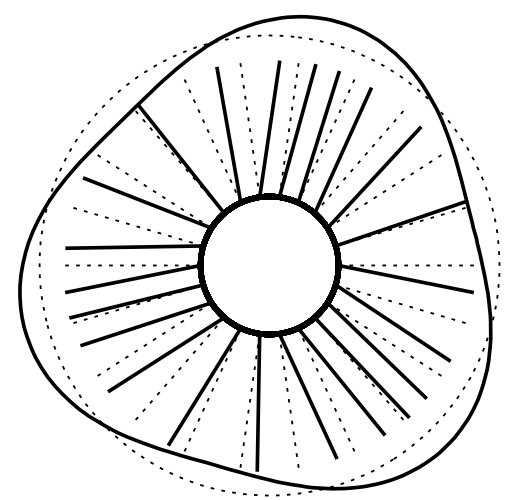

(b) sine modes $u_{s}$ and $\alpha_{s}$

Figure 2: The two 3-nodal diameter modes of the casing and bladed disk

\section{Contact dynamics and explicit time-stepping procedure}

\subsection{Background}

Of particular interest are the contact forces acting between the blade tips and the casing. No friction is considered and, as a consequence, Hamilton's Principle can be used for the derivation of the governing equations, following the procedure detailed in [15]. The so-called impenetrability condition is enforced by the Lagrange multiplier method. In the chosen configuration space, this method has a limited physical interpretation and may be viewed as a mathematical correction of the generalized displacements in order to forbid any residual penetration between the two mechanical components. Hamilton's Principle is written as follows:

$$
\begin{gathered}
\int_{t_{1}}^{t_{2}}\left[\delta \mathrm{T}(\dot{\mathbf{u}}, \dot{\boldsymbol{\alpha}})-\delta \mathrm{V}(\mathbf{u}, \boldsymbol{\alpha})+\delta \mathrm{W}_{n c}(\dot{\mathbf{u}}, \dot{\boldsymbol{\alpha}})+\delta \mathrm{E}_{\lambda}(\mathbf{u}, \boldsymbol{\alpha}, \boldsymbol{\lambda})\right] \mathrm{d} t=0 \\
\forall(\delta \boldsymbol{\alpha}, \delta \mathbf{u}, \delta \boldsymbol{\lambda}) \operatorname{such} \text { as } \delta \boldsymbol{\alpha}\left(t_{1}\right)=\delta \boldsymbol{\alpha}\left(t_{2}\right)=\delta \mathbf{u}\left(t_{1}\right)=\delta \mathbf{u}\left(t_{2}\right)=0
\end{gathered}
$$


The different energies and virtual works involved in the formulation take the following form:

$$
\begin{aligned}
& \mathrm{T}=\frac{1}{2} \dot{\mathbf{u}}^{\mathrm{T}} \mathbf{M}_{c} \dot{\mathbf{u}}+\frac{1}{2} \dot{\boldsymbol{\alpha}}^{\mathrm{T}} \mathbf{M}_{b d} \dot{\boldsymbol{\alpha}} \quad \text { (kinetic energy) } \\
& \mathrm{V}=\frac{1}{2} \mathbf{u}^{\mathrm{T}} \mathbf{K}_{c} \mathbf{u}+\frac{1}{2} \boldsymbol{\alpha}^{\mathrm{T}} \mathbf{K}_{b d} \boldsymbol{\alpha} \quad \text { (potential energy) } \\
& \delta \mathrm{W}_{n c}=-\boldsymbol{\mathbf { \delta }} \mathbf{u}^{\mathrm{T}} \mathbf{D}_{c} \dot{\mathbf{u}}-\boldsymbol{\delta} \boldsymbol{\alpha}^{\mathrm{T}} \mathbf{D}_{b d} \dot{\boldsymbol{\alpha}} \quad \text { (virtual work of dissipative forces) } \\
& \mathrm{E}_{\lambda}=\boldsymbol{\lambda}^{\mathrm{T}} \mathbf{g}(\mathbf{u}, \boldsymbol{\alpha}) \quad \text { (contact energy) }
\end{aligned}
$$

where, in $\boldsymbol{\lambda}$ and $\mathbf{g}$ are respectively stored the Lagrange multipliers and the gap functions between the blade tips and the casing: the latter are detailed in section 4.3 for the present investigation. Structural viscous damping, introduced here by two matrices $\mathbf{D}_{c}$ and $\mathbf{D}_{b d}$, is included in a very general way. It is here added in the mass normalized modal coordinate system through two viscous damping factors $\zeta_{b d}$ and $\zeta_{c}$ such as $d_{b d}=2 \omega_{b d} \zeta_{b d}$ and $d_{c}=2 \omega_{c} \zeta_{c}$ where $\omega_{b d}$ and $\omega_{c}$ are the natural frequency of the chosen $n_{d}$-nodal diameter modes of the bladed disk and casing, respectively. Accordingly, the two damping matrices are simplified such as $\mathbf{D}_{b d}=\operatorname{diag}_{2}\left(d_{b d}\right)$ and $\mathbf{D}_{c}=\operatorname{diag}_{2}\left(d_{c}\right)$. This formulation is discretized in time using the explicit central differences scheme. Denoting by $\mathbf{x}$ the vector storing the generalized displacements of the two structures $\left(\mathbf{x}^{\mathrm{T}}=\left[\boldsymbol{\alpha}^{\mathrm{T}} \mathbf{u}^{\mathrm{T}}\right]\right)$ and by $h$, the time step, vectors in velocity and acceleration become:

$$
\ddot{\mathbf{x}}_{n}=\frac{\mathbf{x}_{n+1}-2 \mathbf{x}_{n}+\mathbf{x}_{n-1}}{h^{2}} \text { et } \quad \dot{\mathbf{x}}_{n}=\frac{\mathbf{x}_{n+1}-\mathbf{x}_{n-1}}{2 h}
$$

This scheme is stable for a very small time step $h$ bounded by the Courant-Friedrichs-Lewy criterion in linear investigations [16]:

$$
\Delta t_{c} \leq \frac{2}{\omega_{\max }}
$$

where $\omega_{\max }$ is the largest eigenvalue of the discretized system. In nonlinear investigations such as those involving contact, an empirical time-step, smaller than for linear cases, has to be chosen.

\subsection{General algorithm for frictionless problems}

Many different algorithms dealing with contact problems in explicit dynamics have been developed over the last few years depending on the type of correction (displacement or velocity) [17]. For both its simplicity and reliability [18], the Forward Increment Lagrange Method developed in [19] is used in our study. This explicit time integration scheme in conjunction with the Lagrange multiplier method handling contact problems is divided into three steps for each time increment. Noting by $\mathbf{M}, \mathbf{D}$ and $\mathbf{K}$, respectively the mass, damping and stiffness matrices of the two structures as a whole, the procedure for a node-to-line contact consists of three main steps:

1. Prediction of the displacements without considering any possible contact. The predicted displacement, denoted with a superscript ${ }^{*}$, is expressed as:

$$
\mathbf{x}^{*}=\left[\frac{\mathbf{M}}{h^{2}}+\frac{\mathbf{D}}{2 h}\right]^{-1}\left(\mathbf{F}_{n}^{\text {ext }}+\left[\frac{2 \mathbf{M}}{h^{2}}-\mathbf{K}\right] \mathbf{x}_{n}+\left[\frac{\mathbf{D}}{2 h}-\frac{\mathbf{M}}{h^{2}}\right] \mathbf{x}_{n-1}\right)
$$

2. Determination of the predicted gap functions between the two structures. Only the negative gap functions, meaning that a penetration has been detected, are stored in $\mathrm{N} \times 1$ vector $\mathbf{g}^{*}$.

3. Correction of the displacements through the calculation of the Lagrange multipliers implying that the detected penetrations vanish:

$$
\mathbf{g}_{n+1}=\mathbf{C}^{\mathrm{T}} \mathbf{x}^{c}+\mathbf{g}^{*}=\mathbf{0}
$$


where the superscript ${ }^{c}$ means that the correction of the displacements is being processed. The contact constraint matrix $\mathbf{C}$ stems from the linear gap expressions between the structures. The new equations of motion taking into account the contact forces along with the contact constraints have to be solved simultaneously:

$$
\begin{aligned}
& {\left[\frac{\mathbf{M}}{h^{2}}+\frac{\mathbf{D}}{2 h}\right] \mathbf{x}^{c}+\mathbf{C} \boldsymbol{\lambda}=\mathbf{0}} \\
& \mathbf{C}^{\mathrm{T}} \mathbf{x}^{c}+\mathbf{g}^{*}=\mathbf{0} \\
& \mathbf{x}_{n+1}=\mathbf{x}^{*}+\mathbf{x}^{c}
\end{aligned}
$$

Eq. (12) can be recast in a more convenient form:

$$
\begin{aligned}
& \boldsymbol{\lambda}=\left[\mathbf{C}^{\mathrm{T}}\left[\frac{\mathbf{M}}{h^{2}}+\frac{\mathbf{D}}{2 h}\right]^{-1} \mathbf{C}\right]^{-1} \mathbf{g}^{*} \\
& \mathbf{x}_{n+1}=\mathbf{x}^{*}-\left[\frac{\mathbf{M}}{h^{2}}+\frac{\mathbf{D}}{2 h}\right]^{-1} \mathbf{C} \boldsymbol{\lambda}
\end{aligned}
$$

\subsection{Modified algorithm}

In our study, the algorithm described above has been slightly modified as follows:

1. Prediction: the displacements of both structures are calculated in modal coordinates. It yields the prediction of four unknowns $u_{c}^{*}, u_{s}^{*}, \alpha_{c}^{*}$ and $\alpha_{s}^{*}$.

2. Determination of the gap functions: each blade angle is deduced from the prediction step thanks to a projection onto the physical coordinates by using Eq. (4). Each gap function $g_{i}^{*}$ can be expressed as:

$$
\mathrm{g}_{i}^{*}=\mathrm{R}_{c}-l_{b}+u_{c}^{*} \cos \beta_{i}^{*}+u_{s}^{*} \sin \beta_{i}^{*} \quad i=1, \mathrm{~N}
$$

where $l_{b}$ is the length of the blades, $\mathrm{R}_{c}$, the radius of the casing and $\beta_{i}^{*}=n_{d}\left(\Omega t+\Phi_{i}+\alpha_{i}^{*}\right)$ as illustrated in Fig. 3. $\Phi_{i}$ is the initial angle of the $i$-th blade in a stationary frame.

3. Correction step: Lagrange multipliers are calculated so as to eliminate the negative gap between the guessed penetrating blades and the targeted casing. The linearization of the $i$-th gap function between the prediction step and the correction step yields:

$$
\mathrm{g}_{i, n+1}=\mathrm{g}_{i}^{*}+u_{c}^{c} \cos \beta_{i}^{*}+u_{s}^{c} \sin \beta_{i}^{*}+n_{d}\left(u_{s}^{*} \cos \beta_{i}^{*}-u_{c}^{*} \sin \beta_{i}^{*}\right) \alpha_{i}^{c}=0 \quad i=1, \mathrm{~N}
$$

As an illustration and for the sake of brevity, let us consider a bladed disk with five blades from which two (number 1 and 2) are in contact with the casing during the on-going timestep. From Eq. (15), Eq. (12b) takes the form:

$$
\left[\begin{array}{ccccccc}
\cos \beta_{1}^{*} & \sin \beta_{1}^{*} & \mathrm{~A}_{1}^{*} & 0 & 0 & 0 & 0 \\
\cos \beta_{2}^{*} & \sin \beta_{2}^{*} & 0 & \mathrm{~A}_{2}^{*} & 0 & 0 & 0 \\
\cos \beta_{3}^{*} & \sin \beta_{3}^{*} & 0 & 0 & \mathrm{~A}_{3}^{*} & 0 & 0 \\
\cos \beta_{4}^{*} & \sin \beta_{4}^{*} & 0 & 0 & 0 & \mathrm{~A}_{4}^{*} & 0 \\
\cos \beta_{5}^{*} & \sin \beta_{5}^{*} & 0 & 0 & 0 & 0 & \mathrm{~A}_{5}^{*}
\end{array}\right]\left(\begin{array}{l}
u_{c}^{c} \\
u_{s}^{c} \\
\alpha_{1}^{c} \\
\alpha_{2}^{c} \\
\alpha_{3}^{c} \\
\alpha_{4}^{c} \\
\alpha_{5}^{c}
\end{array}\right)+\left(\begin{array}{c}
\mathrm{g}_{1}^{*} \\
\mathrm{~g}_{2}^{*} \\
0 \\
0 \\
0
\end{array}\right)=\left(\begin{array}{l}
0 \\
0 \\
0 \\
0 \\
0
\end{array}\right)
$$

where $\mathrm{A}_{i}^{*}=n_{d}\left(u_{s}^{*} \cos \beta_{i}^{*}-u_{c}^{*} \sin \beta_{i}^{*}\right)$.

Finally, displacements are projected back to the modal coordinates, time is incremented and a new prediction is calculated. 


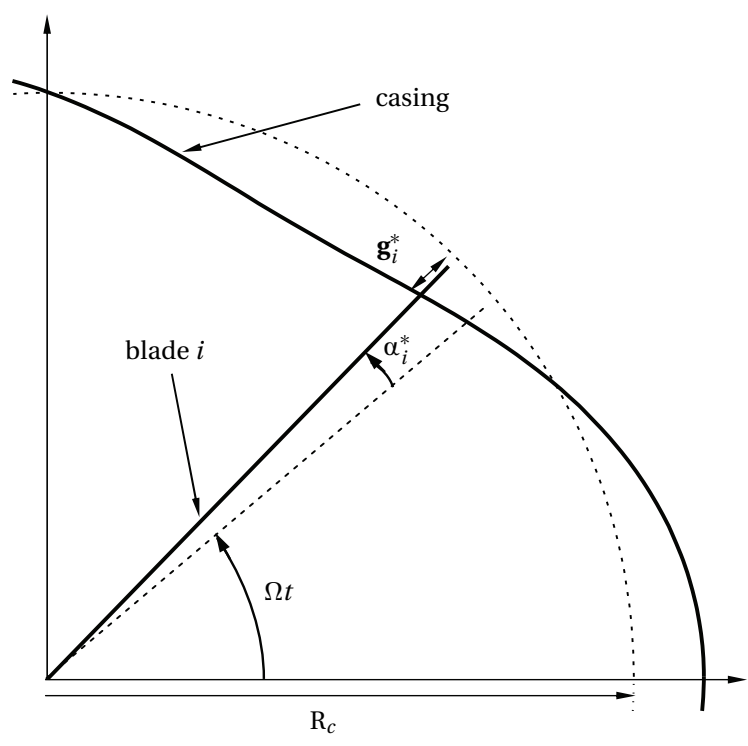

Figure 3: Schematic of the $i$-th predicted gap distance between blade $i$ and casing

\subsection{Numerical simulations and results}

Numerical simulations are performed with respect to the rotational velocity of the bladed disk $\Omega$ over the $0-500 \mathrm{rad} / \mathrm{s}$ running speed range. Contact between the rotor and the stator is initiated by a $50 \mu$ s forcing pulse applied on the modal amplitude $u_{c}$ at the very beginning of the each simulation for which the rotational velocity $\Omega$ is constant. The rotor of the chosen model is perfectly balanced and no eccentricity between the two structures is introduced. The other mechanical parameters used in this study are listed in Table 1: $m_{b}$ and $m_{c}$ are not normalized as their physical value is required in the contact algorithm.

Table 1: Mechanical parameters of the model

\begin{tabular}{lll}
\hline bladed disk & casing & other \\
\hline$l_{b}=0.4999 \mathrm{~m}$ & $\mathrm{R}_{c}=0.5 \mathrm{~m}$ & $n_{d}=3$ or 4 \\
$m_{b}=0.21 \mathrm{~kg}$ & $m_{c}=2 \mathrm{~kg}$ & $\mathrm{~N}=22$ \\
$k_{b}=15,000 \mathrm{~N} / \mathrm{m}$ & $k_{c}=1,544,000 \mathrm{~N} / \mathrm{m}$ & $\Delta t_{c}=2.27 \cdot 10^{-3} \mathrm{~s}$ \\
$k_{b b}=3,000 \mathrm{~N} / \mathrm{m}$ & $\zeta_{c}=0.05$ & $\Omega_{c}\left(n_{d}=3\right)=292.87 \mathrm{rad} / \mathrm{s}$ \\
$\zeta_{b d}=0.04$ & $\omega_{c}=878.63 \mathrm{rad} / \mathrm{s}$ & $\Omega_{c}\left(n_{d}=4\right)=256.12 \mathrm{rad} / \mathrm{s}$ \\
$\omega_{b d}=286.3 \mathrm{rad} / \mathrm{s}$ & & \\
\hline
\end{tabular}

\subsubsection{Time-step size convergence analysis}

It is well-known that strong nonlinearities such as direct structural contact play an important role on the time-step size of an explicit algorithm [20]. The linearization of the gap functions and more importantly, the modal displacement field used in this study restrict the time step to very small values. To determine an appropriate size of the latter, numerical tests with several different time steps, $h=10^{-5} \mathrm{~s}, h=10^{-6} \mathrm{~s}$ and $h=10^{-7} \mathrm{~s}$ have been conducted. The respective results are depicted in Fig. 4. It is shown that the time-step convergence is difficult to achieve. Decreasing the time step to $h=10^{-8}$ s similarly leads to a phase shift in comparison to the displayed results. As a matter of fact, due to the two degree-of-freedom structural modal description of the system, local information travels with an infinite velocity from one part of the structures to another one and consequently, the contact detection technique is facing difficulties in the estimation of the correct gap functions. Nevertheless, for a steady-state with repetitive to permanent contact between the two structures, 


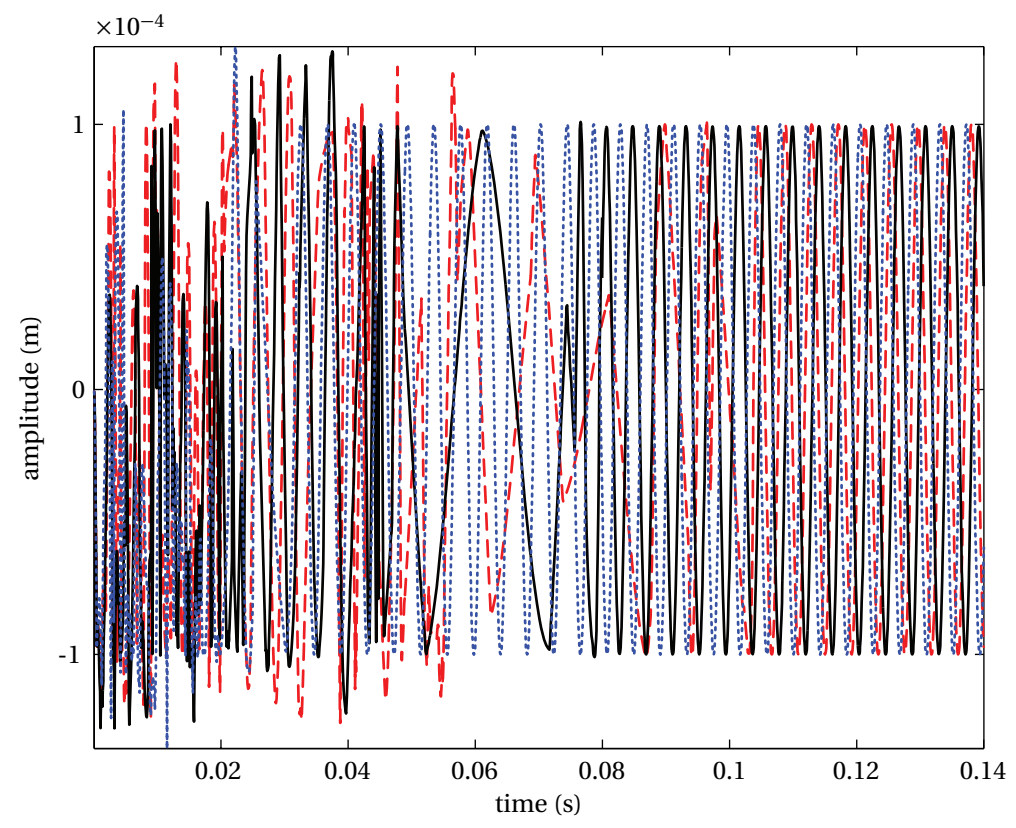

Figure 4: Time evolution of $u_{c}$ during interaction for $h=10^{-6} \mathrm{~s}(\cdots), h=5 \cdot 10^{-7} \mathrm{~s}(--)$ and $h=$ $10^{-7} \mathrm{~s}(-)$ for $n_{d}=3$ and $\Omega=500 \mathrm{rad} / \mathrm{s}$

the qualitative general behavior of the mechanical system remains identical, as seen in Fig. 4 for $t>0.04 \mathrm{~s}$. Therefore, it has been decided in an almost-empirical manner to keep $h=10^{-7} \mathrm{~s}$ as the time-step in our algorithm, time-step that can be compared to the Courant critical time step $\Delta t_{c}$ given in Table 1. A focus on the distances between the blade tips and the casing depicted in Fig. 5 confirms that the contact algorithm is successful: gap distances are all non-negative and the algorithm remains stable for all times.

\subsubsection{Response of the structures}

Based on an extensive numerical simulation study along $\Omega$ and the amplitude of the pulse applied on the casing, two types of response are observed. The first one is characterized by several impacts between the two structures at the beginning of the simulation, then followed by a decrease of the vibration amplitudes to zero due to structural damping. This response is not of interest in this study. In contrast, an exchange of energy between the stator and the rotor occurs in the second type of behavior. This interesting case features a steady-state where the blades no longer vibrate while two of them (with the chosen parameters listed in Tab. 1) are in permanent contact with the casing.

They are highlighted by solid and dashed bold black lines in Fig. 5. During this permanent contact steady-state, these two blades push a traveling wave that propagates in the casing at the angular velocity of the bladed disk as illustrated in Fig. 6(a): projected back to the physical space, the two depicted curves represent a forward traveling wave starting at $t \simeq 0.08 \mathrm{~s}$. On the other hand, Fig. $6(\mathrm{~b})$ shows that the bladed disk reaches a permanent static-only deformation state after the transient response has died away. This new phenomenon is referred to as locked motion and is studied in detail in the sequel. The results given below remain very general and can be extended to any kind of situation as illustrated in Figs. 7(a) and 7(b) for $n_{d}=4$ where the same kind of behavior is observed. The fact that the locked motion starts at $t \simeq 0.08 \mathrm{~s}$ for both $n_{d}=3$ and $n_{d}=4$ is a coincidence. Also, it is worth noting that the number of blades simultaneously in contact with the casing mainly depends on the parameters $n_{d}, \mathrm{~N}$ as well as the initial clearance between the two structures. Referring to Fig. 5 where blades \#6 and \#21 are in permanent contact with the casing, other configurations involving different blades were detected. This is mainly due to the inherent nonlinear nature of the 


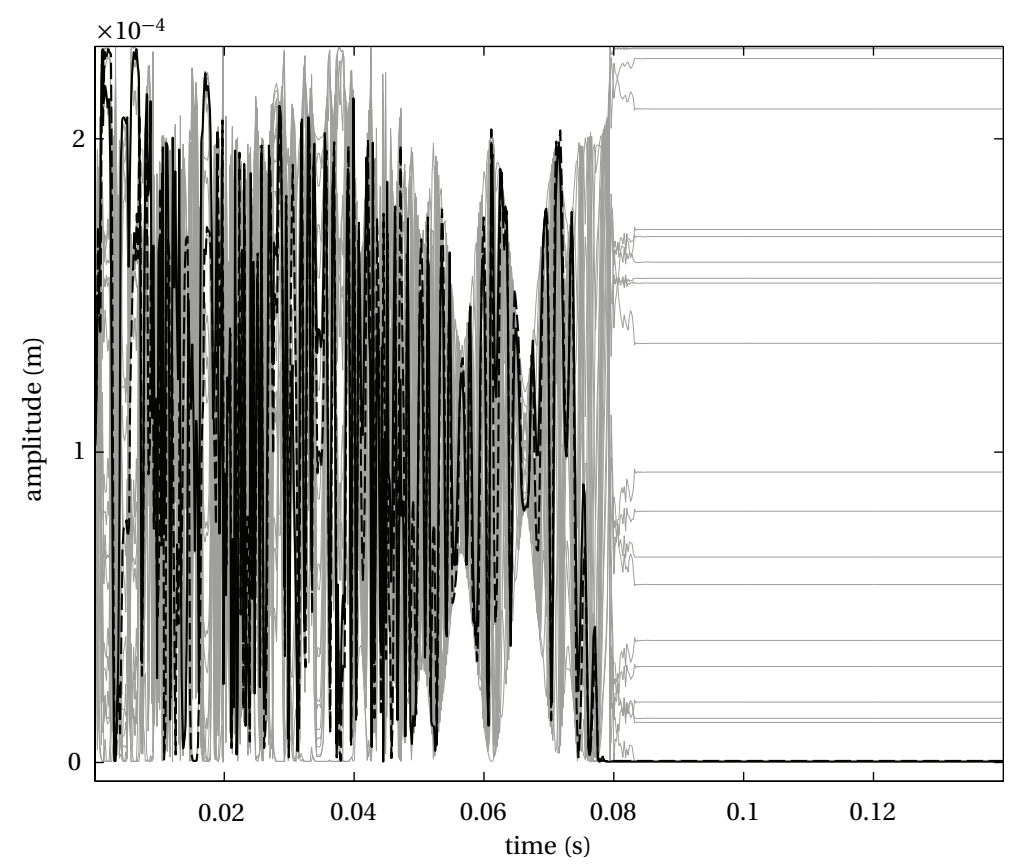

Figure 5: Distances between the casing and the 22 blade tips for $n_{d}=3$ and $\Omega=500 \mathrm{rad} / \mathrm{s}$ : two blades \#6 (---) and \#21 (-) are in permanent contact with the casing during the interaction phenomenon

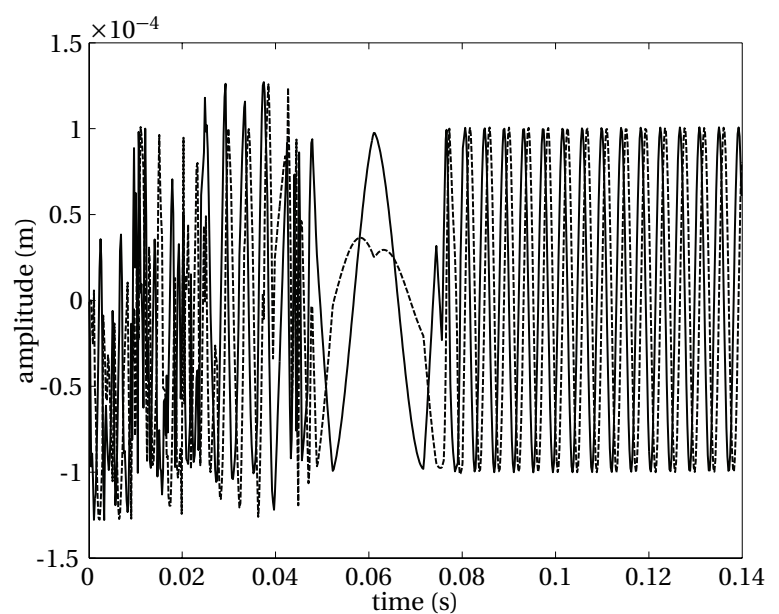

(a) casing: $u_{c}(-)$ and $u_{s}(---)$

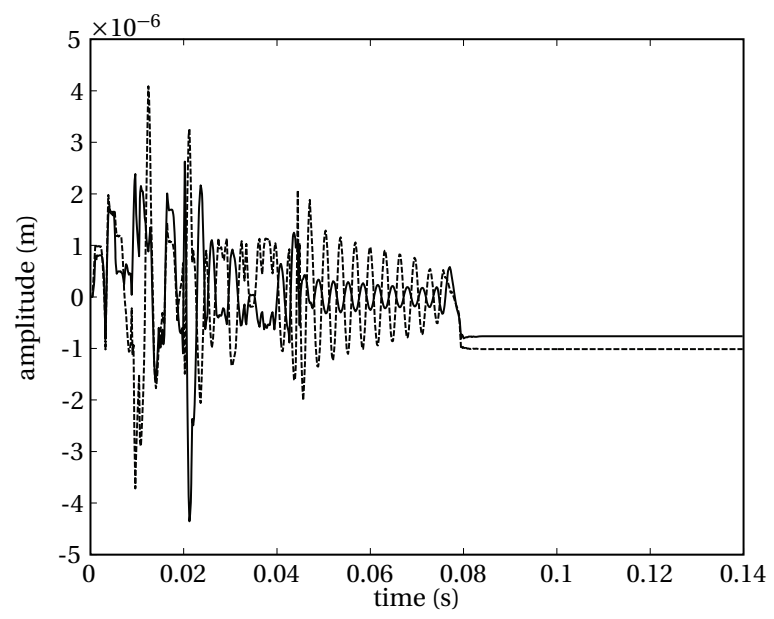

(b) bladed disk: $\alpha_{c}(-)$ and $\alpha_{s}(---)$

Figure 6: Modal vibrations of the structures for $\Omega=500 \mathrm{rad} / \mathrm{s}$ and $n_{d}=3$ : at $t \simeq 0.08 \mathrm{~s}$, a $n_{d}$ nodal diameter forward traveling wave starts to propagate in the casing while the blade disk simultaneously reaches a purely static deformation state

system, which is not explored in detail in the present investigation.

\subsubsection{Conditions of interaction}

It appears that several parameters (rotational velocity, initial forcing pulse amplitude, structural damping...), and the combination thereof, play a key role on the occurrence of the locked motion. Concerning the rotational velocity and the initial forcing pulse amplitude, Fig. 8 reveals a zone of possible interaction for $\Omega \geq \omega_{c} / n_{d}$. The initial forcing pulse in this zone must be sufficiently large 


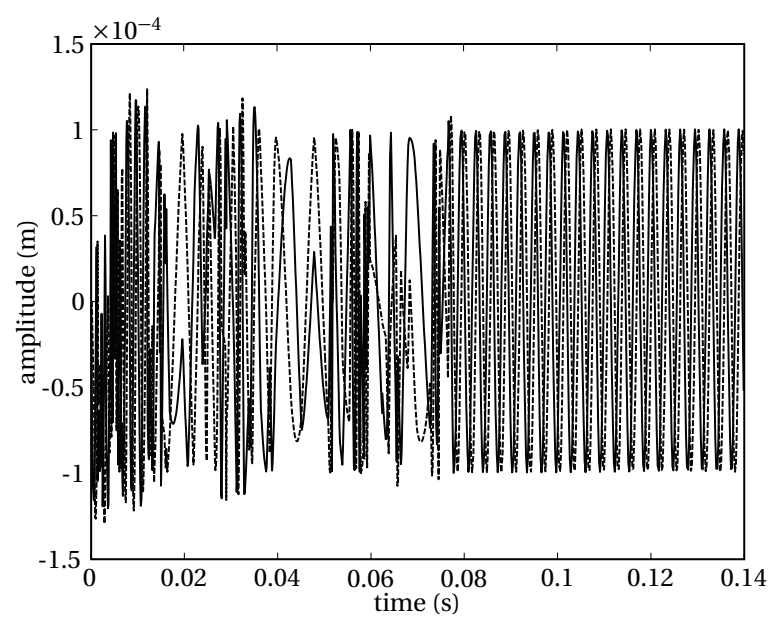

(a) casing: $u_{c}(-)$ and $u_{s}(---)$

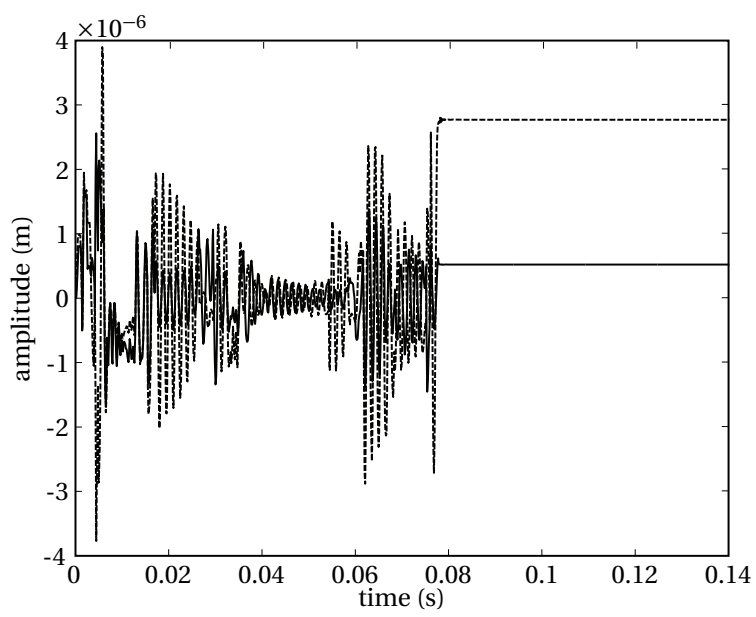

(b) bladed disk: $\alpha_{c}(-)$ and $\alpha_{s}(---)$

Figure 7: Modal vibrations of the structures for $\Omega=500 \mathrm{rad} / \mathrm{s}$ and $n_{d}=4$ : at $t \simeq 0.08 \mathrm{~s}$, a $n_{d}$ nodal diameter forward traveling wave starts to propagate in the casing while the blade disk simultaneously reaches a purely static deformation state

to initiate the interaction phenomenon. Below $\omega_{c} / n_{d}$ and for any pulse amplitude, a locked motion could not be detected and seems impossible. This leads to the definition of a critical velocity $\Omega_{c}$ of the investigated system:

$$
\Omega_{c}=\frac{\omega_{c}}{n_{d}}
$$

Although the few previous findings have shown the importance of the natural frequency of the bladed disk $\omega_{b d}$ (see Eq. (31) and [10]), Fig. 6(b) demonstrates that the role of this structure in the phenomenon vanishes because of its permanent static-only deformation state behavior during the interaction. However, a clear conclusion on the time integration results is premature due to the high sensitivity of the method to the time step size and to the external forcing conditions. Accordingly, a frequency-domain approach is now proposed for its capability to provide a more intrinsic description of the system behavior that naturally complements a time integration investigation.

\section{Frequency-domain study of the 'locked-motion'}

In order to avoid the high sensitivity of the results to the initial conditions, to the amplitude of the initial forcing pulse and to the time step size, the Harmonic Balance Method (HBM) is used. This frequency-domain method is capable of predicting efficiently the steady-state periodic behavior of strongly nonlinear mechanical systems [21]. The HBM method allows for parameter studies that would carry prohibitive cost using direct time integration, and results in a better physical understanding of the solutions. It is used in the present study in order to complement the time integration results and demonstrate in an almost analytical manner that the locked-motion only occurs for a specific range of rotational velocities.

\subsection{Governing equations}

The results of the previous section are used for the derivation of the governing equations. Without loss of generality, it is considered here that only two blades, denoted by $i_{1}$ and $i_{2}$, are in permanent contact with the casing. The resulting equations for the casing take the following form where 


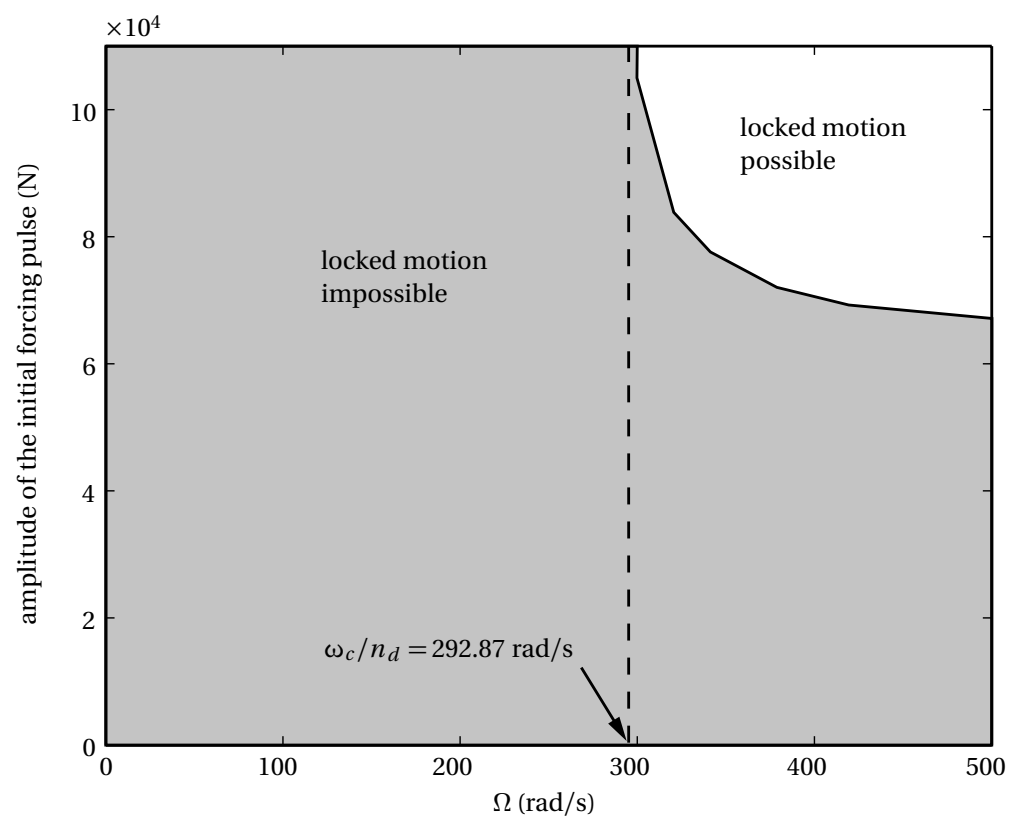

Figure 8: Zone of interaction in the 'Initial Pulse Amplitude - Rotational Velocity $\Omega$ ' space

damping terms have been dismissed as there is no transient response to be damped:

$$
\left\{\begin{array}{l}
m_{c} \ddot{u}_{c}+k_{c} u_{c}+\sum_{j=1}^{2} \lambda_{j} \cos \beta_{i_{j}}=0 \\
m_{c} \ddot{u}_{s}+k_{c} u_{s}+\sum_{j=1}^{2} \lambda_{j} \sin \beta_{i_{j}}=0
\end{array}\right.
$$

where $\beta_{i_{j}}$ is defined in Eq. (14). Concerning the bladed disk and referring to the previous conclusions, only static terms are considered. Accordingly, equations associated with the motion of the two blades in permanent contact with the casing become:

$$
\left(k_{b}+2 k_{b b}\right) \alpha_{i_{j}}-k_{b b}\left(\alpha_{i_{j}-1}+\alpha_{i_{j}+1}\right)+\lambda_{j} n_{d}\left(u_{s} \cos \beta_{i_{j}}-u_{c} \sin \beta_{i_{j}}\right)=0, \quad j=1,2
$$

where $\alpha_{i_{j}-1}$ and $\alpha_{i_{j}+1}$ are deduced from $\alpha_{i_{j}}$, for $j=1,2$ using Eq. (4):

$$
\left(\begin{array}{l}
\alpha_{i_{1}-1} \\
\alpha_{i_{1}+1} \\
\alpha_{i_{2}-1} \\
\alpha_{i_{2}+1}
\end{array}\right)=\left[\begin{array}{ll}
\mathbf{p}_{\mathbf{c}}\left(i_{1}-1\right) & \mathbf{p}_{\mathbf{s}}\left(i_{1}-1\right) \\
\mathbf{p}_{\mathbf{c}}\left(i_{1}+1\right) & \mathbf{p}_{\mathbf{s}}\left(i_{1}+1\right) \\
\mathbf{p}_{\mathbf{c}}\left(i_{2}-1\right) & \mathbf{p}_{\mathbf{s}}\left(i_{2}-1\right) \\
\mathbf{p}_{\mathbf{c}}\left(i_{2}+1\right) & \mathbf{p}_{\mathbf{s}}\left(i_{2}+1\right)
\end{array}\right]\left[\begin{array}{ll}
\mathbf{p}_{\mathbf{c}}\left(i_{1}\right) & \mathbf{p}_{\mathbf{s}}\left(i_{1}\right) \\
\mathbf{p}_{\mathbf{c}}\left(i_{2}\right) & \mathbf{p}_{\mathbf{s}}\left(i_{2}\right)
\end{array}\right]^{-1}\left(\begin{array}{c}
\alpha_{i_{1}} \\
\alpha_{i_{2}}
\end{array}\right)
$$

Finally, it is necessary to add the two permanent contact conditions between blades $i_{1}, i_{2}$ and the casing:

$$
\mathrm{R}_{c}-l_{b}+u_{c} \cos \beta_{i_{j}}+u_{s} \sin \beta_{i_{j}}=0, \quad j=1,2
$$

This formulation results in a nonlinear differential system of six equations and six unknowns $u_{c}, u_{s}$, $\lambda_{1}, \lambda_{2}, \alpha_{i_{1}}, \alpha_{i_{2}}$ that cannot be solved analytically.

\subsection{Solution method}

Time integration has shown pure harmonic (casing) and static (bladed disk) responses. Seeking the existence of periodic solution to the assumed configuration induces the use of the Harmonic 
Balance Method (HBM). As a matter of fact, this is a classical technique for studying nonlinear steady state oscillations. The response as well as the nonlinear restoring forces are expanded in Fourier series. In certain conditions with no explicit external forcing, HBM can provide non-zero solutions that can be seen as nonlinear normal modes of the system, namely an intrinsic description of the behavior of the system. Moreover, HBM transforms a nonlinear differential system of equations in a nonlinear algebraic system of equations that can be easier to solve.

The general idea of the HBM is to represent each time history by its frequency content and solve through an iterative procedure the set of equations obtained by balancing the terms with the same frequency component [22]. Equations of motion including contact forces and keeping notations of Eq. (10) can be set as:

$$
\mathbf{M} \ddot{\mathbf{x}}+\mathbf{K x}+\mathbf{C} \boldsymbol{\lambda}=\mathbf{F}^{\mathrm{ext}}
$$

where $\mathbf{F}^{\text {ext }}$ stands for the external periodic excitation and $\boldsymbol{\lambda}$ represents the nonlinear contact forces. As mentioned earlier, damping is not considered in Eq. (22) and in the remainder because it is not a parameter of interest in this work. Although it may be included in time marching procedures to quickly reach the steady state response, it may be neglected in frequency-domain techniques. Next, assuming a steady-state periodic response, the generalized displacements are expanded in the frequency-domain as a Fourier series up to $\mathrm{N}_{h}$ temporal harmonics:

$$
\mathbf{x}(t)=\sum_{k=0}^{\mathrm{N}_{h}} \mathbf{x}_{k}^{c} \cos (k \omega t)+\sum_{k=1}^{\mathrm{N}_{h}} \mathbf{x}_{k}^{s} \sin (k \omega t)
$$

Then using Eq. (23) and balancing the sin and cos terms, Eq. (22) can be recast in the following form:

$$
\Lambda \tilde{\mathbf{x}}+\tilde{\boldsymbol{\lambda}}=\tilde{\mathbf{F}}^{\mathrm{ext}}
$$

where:

$$
\boldsymbol{\Lambda}=\left[\begin{array}{cc}
\mathbf{K} & \mathbf{0} \\
\mathbf{0} & \boldsymbol{\Lambda}_{h}
\end{array}\right] \text { and } \boldsymbol{\Lambda}_{h, k}=\left[\begin{array}{cc}
-(k \omega)^{2} \mathbf{M}+\mathbf{K} & \mathbf{0} \\
\mathbf{0} & -(k \omega)^{2} \mathbf{M}+\mathbf{K}
\end{array}\right], \quad k=1, \ldots, \mathbf{N}_{h}
$$

The $k$-th block $\boldsymbol{\Lambda}_{h, k}$ defines the dynamic stiffness matrix for the $k$-th harmonic. Accordingly, $\tilde{\mathbf{x}}, \tilde{\lambda}$ and $\tilde{\mathbf{F}}^{\text {ext }}$ are frequency domain counterparts of time domain $\mathbf{x}, \mathbf{C} \boldsymbol{\lambda}$ and $\mathbf{F}^{\text {ext }}$, respectively. Several different methods allow for taking into account the contact forces that are evaluated in the time-domain during resolution. In [23], the displacements are directly corrected with respect to the inequality contact constraints without calculating the contact forces whereas [24] requires the expression of the contact forces in the frequency-domain in order to solve Eq. (24).

Based on the time-domain results the following is assumed:

- a single harmonic at frequency $n_{d} \Omega$ describes the casing dynamics during interaction:

$$
\left\{\begin{array}{l}
u_{c}(t)=u_{c c} \cos \left(n_{d} \Omega t\right)+u_{c s} \sin \left(n_{d} \Omega t\right) \\
u_{s}(t)=u_{s c} \cos \left(n_{d} \Omega t\right)+u_{s s} \sin \left(n_{d} \Omega t\right)
\end{array}\right.
$$

- the two contact forces are constant. Consequently, only the 0 -th order terms $\lambda_{1}$ and $\lambda_{2}$ (see Eq. (18)) of the Fourier series are accounted for.

- the two bladed disk modal amplitudes are constant. Consequently, only the 0-th order terms $\alpha_{i 1}$ and $\alpha_{i 2}$ (see Eq. (20)) of the Fourier series are accounted for.

A projection of Eqs. (18), (19) and (21) onto the basis formed by the functions [1, $\left.\cos \left(n_{d} \Omega t\right), \sin \left(n_{d} \Omega t\right)\right]$ followed by an integration over the domain $t=\left[1,2 \pi /\left(n_{d} \Omega\right)\right]$ (Galerkin procedure) leads to a nonlinear algebraic system of eight equations and eight unknowns $u_{c c}, u_{c s}, u_{s c}, u_{s s}, \lambda_{1}, \lambda_{2}, \alpha_{i_{1}}$ and 
$\alpha_{i_{2}}$. Because of the very small amplitudes of displacement exhibited by the bladed disk during the interaction phenomenon, the following first order expansions are considered:

$$
\left\{\begin{array}{l}
\cos \left(n_{d} \alpha_{i_{j}}\right) \simeq 1 \\
\sin \left(n_{d} \alpha_{i_{j}}\right) \simeq n_{d} \alpha_{i_{j}}
\end{array}, \quad j=1,2\right.
$$

By denoting $c_{1}=\cos \left(n_{d} \Phi_{i_{1}}\right), c_{2}=\cos \left(n_{d} \Phi_{i_{2}}\right), s_{1}=\sin \left(n_{d} \Phi_{i_{1}}\right)$ and $s_{2}=\sin \left(n_{d} \Phi_{i_{2}}\right)$, the nonlinear system of equations can be written in the following manner:

$$
\left\{\begin{array}{c}
\lambda_{1} n_{d} s_{1}\left(u_{s s}+u_{c c}+n_{d} \alpha_{i_{1}}\left(u_{s c}-u_{c s}\right)\right)+\lambda_{1} n_{d} c_{1}\left(u_{c s}-u_{s c}+n_{d} \alpha_{i_{1}}\left(u_{s s}+u_{c c}\right)\right) \\
+\left(k_{b}+2 k_{b b}\right) \alpha_{i_{1}}-k_{b b}\left(\alpha_{i_{1}-1}+\alpha_{i_{1}+1}\right)=0 \\
\lambda_{2} n_{d} s_{2}\left(u_{s s}+u_{c c}+n_{d} \alpha_{i_{2}}\left(u_{s c}-u_{c s}\right)\right)+\lambda_{2} n_{d} c_{2}\left(u_{c s}-u_{s c}+n_{d} \alpha_{i_{2}}\left(u_{s s}+u_{c c}\right)\right) \\
+\left(k_{b}+2 k_{b b}\right) \alpha_{i_{2}}-k_{b b}\left(\alpha_{i_{2}-1}+\alpha_{i_{2}+1}\right)=0 \\
2\left(\mathrm{R}_{c}-l_{b}\right)+s_{1}\left(u_{s c}-u_{c s}-n_{d} \alpha_{i_{1}}\left(u_{s s}+u_{c c}\right)\right)+c_{1}\left(u_{s s}+u_{c c}+n_{d} \alpha_{i_{1}}\left(u_{s c}-u_{c s}\right)\right)=0 \\
2\left(\mathrm{R}_{c}-l_{b}\right)+s_{2}\left(u_{s c}-u_{c s}-n_{d} \alpha_{i_{2}}\left(u_{s s}+u_{c c}\right)\right)+c_{2}\left(u_{s s}+u_{c c}+n_{d} \alpha_{i_{2}}\left(u_{s c}-u_{c s}\right)\right)=0 \\
\left(k_{c}-n_{d}^{2} \Omega^{2} m_{c}\right) u_{c c}+\lambda_{1}\left(c_{1}-n_{d} \alpha_{i_{1}} s_{1}\right)+\lambda_{2}\left(c_{2}-n_{d} \alpha_{i_{2}} s_{2}\right)=0 \\
\left(k_{c}-n_{d}^{2} \Omega^{2} m_{c}\right) u_{c s}-\lambda_{1}\left(s_{1}+n_{d} \alpha_{i_{1}} c_{1}\right)-\lambda_{2}\left(s_{2}+n_{d} \alpha_{i_{2}} c_{2}\right)=0 \\
\left(k_{c}-n_{d}^{2} \Omega^{2} m_{c}\right) u_{s c}+\lambda_{1}\left(s_{1}+n_{d} \alpha_{i_{1}} c_{1}\right)+\lambda_{2}\left(s_{2}+n_{d} \alpha_{i_{2}} c_{2}\right)=0 \\
\left(k_{c}-n_{d}^{2} \Omega^{2} m_{c}\right) u_{s s}+\lambda_{1}\left(c_{1}-n_{d} \alpha_{i_{1}} s_{1}\right)+\lambda_{2}\left(c_{2}-n_{d} \alpha_{i_{2}} s_{2}\right)=0
\end{array}\right.
$$

The solution vector is obtained iteratively by a nonlinear procedure such as the quasi-Newton method implemented in Matlab.

\subsection{Results}

The numerical model outlined in the previous section was implemented in a computer code and simulations of the proposed technique were carried out using $\Omega$ as a parameter. It is now assumed that $n_{d}=3, i_{1}=6$ and $i_{2}=21$. The results confirm that $\Omega_{c}$ is a specific rotational velocity of this system for which the sign of the Lagrange multipliers changes, as depicted in Fig. 9 (note that $\lambda_{1}=$ $\lambda_{2}$ ). Similarly, the bladed disk continuously goes from one steady-state to another while crossing this value as Fig. 10(b) indicates. However, the behavior of the casing is $\Omega$-independent as illustrated in Fig. 10(a) and the harmonic amplitudes are consistent with a forward traveling wave propagating in the casing since they induce the following displacement field:

$$
\left\{\begin{array}{l}
u_{c}(t)=u_{c c} \cos \left(n_{d} \Omega t\right)+u_{c s} \sin \left(n_{d} \Omega t\right) \\
u_{s}(t)=-u_{c s} \cos \left(n_{d} \Omega t\right)+u_{c c} \sin \left(n_{d} \Omega t\right)
\end{array}\right.
$$

Furthermore, the sinusoidal wave amplitudes observed in Fig. 6(a) are reproduced with the present approach showing a perfect agreement between the two methods and the consistency of the assumptions used for the HBM derivation. Nevertheless, the solution is physically unacceptable when $\Omega<\omega_{c} / n_{d}$ while numerically correct. In simple terms, the well-known Signorini inequality conditions arising in contact mechanics [25] here collapse into the same equality condition given in Eq. (21) because of the explained assumptions. Mathematically, condition (21) affects non-admissible gap functions as well as admissible gap functions, which is not correct: additional simple calculations on the contact forces when $\Omega<\omega_{c} / n_{d}$ reveal that the casing pushes the bladed disk, a nonphysical configuration that has to be dismissed. This main result is fully consistent with the presupposed interaction zone found with the time integration approach and depicted in Fig. 8: the locked-motion is unreachable for $\Omega \leq \omega_{c} / n_{d}$ under any initial forcing pulse applied on the casing. 


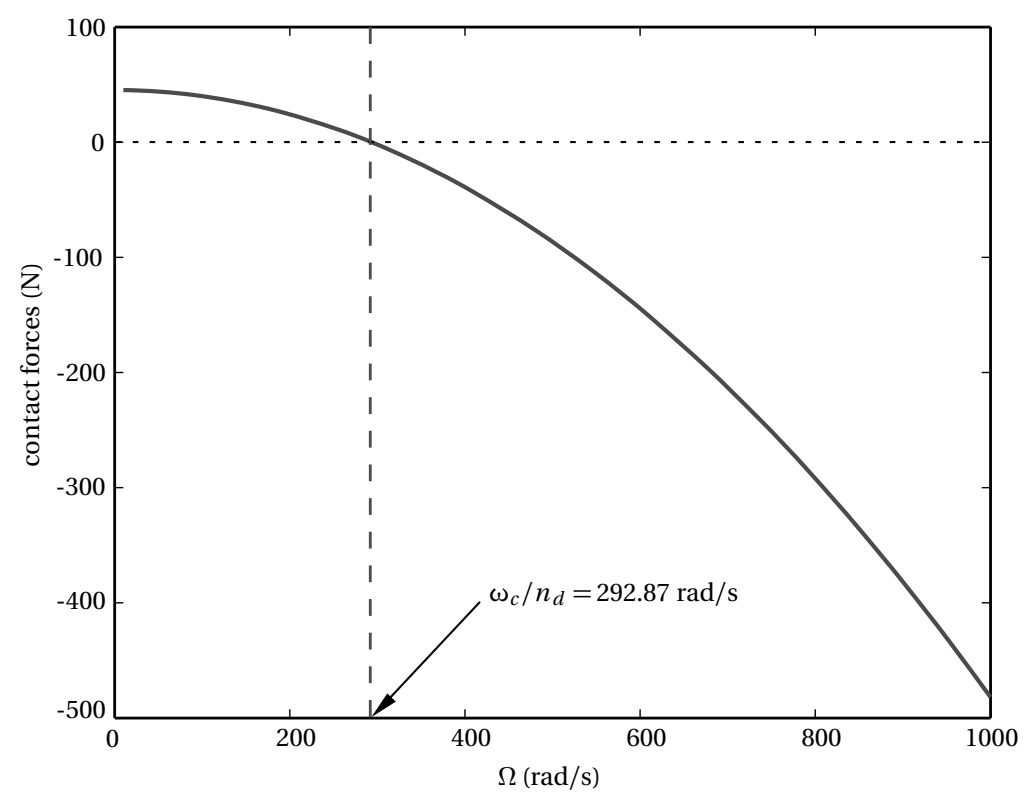

Figure 9: Contact forces $\lambda_{1}$ and $\lambda_{2}$ versus $\Omega$

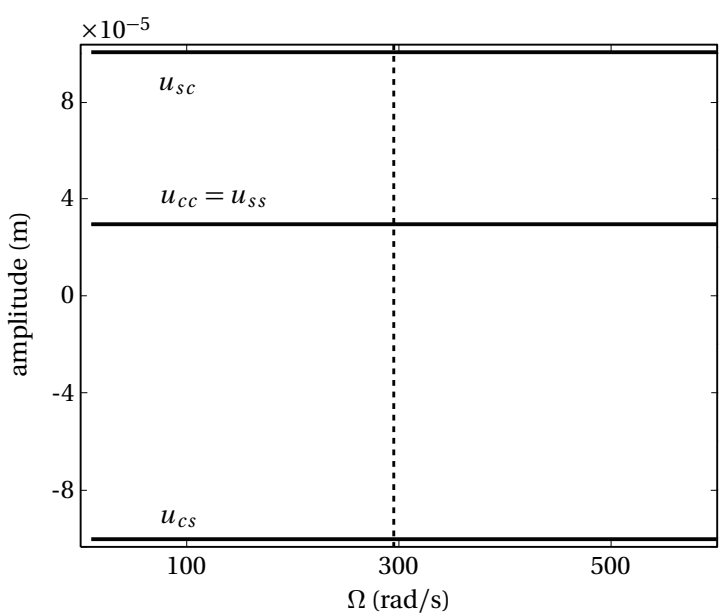

(a) casing

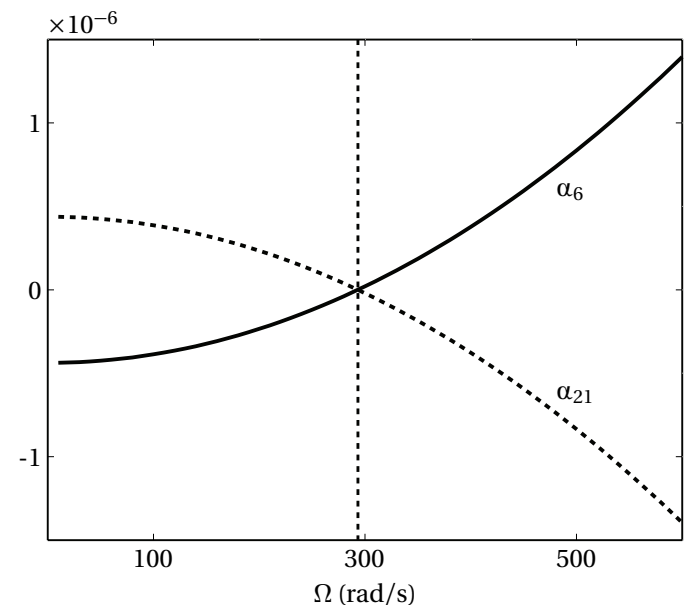

(b) bladed disk

Figure 10: Frequency-domain amplitudes of both structures with respect to $\Omega$

The introduction of an engine-order excitation has no noticeable effects on the results. A discussion about time consumption between the two methods is meaningless considering that the HBM outputs a semi-analytical, comprehensive result compared to the numerical investigation of the time integration. Nevertheless a CPU estimation can be given: the comparison is highly favorable to HBM that is around a thousand times faster than the time integration procedure. Furthermore, analogously to the time integration investigation, the nonlinear nature of Eq. (27) gives rise to multiple admissible solutions where different blades (restricted to two in the present formulation) are in permanent contact with the casing. Situations with more blades in permanent contact were not explored but are obviously conceivable.

\section{Summary and concluding remarks}

The emphasis of the study has been placed on the understanding of the modal interaction phenomenon that can occur between a bladed disk and an outer casing. The mechanical model associ- 
ated with the developed numerical tool is extremely simple. The bladed disk is constituted of rigid bodies, representing the blades, connected together and to the disk by linear springs. This structure is then reduced to its two $n_{d}$-nodal diameter modes. The casing is directly discretized on its two $n_{d}$-nodal diameter modes. Both solution methods, an explicit time marching procedure and a frequency-domain approach, are in really good agreement in the prediction of an interaction phenomenon occurring only if the following condition is met:

$$
\Omega \geq \omega_{c} / n_{d}
$$

Equation (29) means that the rotational velocity of the bladed disk $\Omega$ must be greater than the natural traveling wave speed of the $n_{d}$-nodal diameter mode of the casing so that an interaction can be detected. It is worth mentioning that this kind of interaction can only be investigated with flexible structures allowing for multiple simultaneous contact locations.

Nevertheless, considering the theory presented in section 2, if both structures vibrate at their own natural frequencies, four cases of traveling wave speed coincidence can be stated for $\Omega$ :

$$
\pm \omega_{c}=n_{d} \Omega \pm \omega_{b d}
$$

Taking into account physical considerations on the direction of the contact and friction forces between the two structures - forward on the casing and backward on the bladed disk if the rotor rotates counter-clockwise -, only one of these equations can be considered as dangerous:

$$
\omega_{c}=n_{d} \Omega-\omega_{b d}
$$

At this speed, both structures are driven in resonance by the contact forces. This rotational velocity must be avoided because resonance can cause undesired large amplitude vibrations.

The study undertaken here is the first step in understanding the interaction phenomena that can occur in an aircraft-engine and precedes the development of an improved model able to capture the type of phenomenon mentioned above. Indeed, future roads of investigation are numerous. Currently under consideration is a theoretical research work with a model involving a smooth differentiable contact law. Existence, stability and bifurcation analyses are conducted with the following improvements:

1. A priori uncoupled flexural and axial displacement directions.

2. Linearly coupled flexural and axial displacement directions (initially curved beam for instance) so that the modeshapes of the casing and bladed disk can geometrically match.

3. Displacement fields with different nodal diameter modes.

\section{Acknowledgement}

Thanks go to Snecma for its technical and financial support. This work takes place in the framework of the MAIA mechanical research and technology program sponsored by CNRS, ONERA and SAFRAN Group.

\section{References}

[1] C.-W. Lee, Vibration Analysis of Rotors, Kluwer Academic Publishers, 1993.

[2] D. Childs, Turbomachinery Rotordynamics Phenomena, Modeling and Analysis, Prentice Hall, 1993. 
[3] J. M. Vance, Rotordynamics of Turbomachinary, Wiley-Interscience, 1987.

[4] Y. Choi, Investigation on the whirling motion of full annular rotor rub, Journal of Sound and Vibration 258 (1) (2002) 191-198.

http://dx.doi.org/10.1006/jsvi.2002.5091

[5] A. Muszynska, P. Goldman, Chaotic responses of unbalanced rotor/bearing/stator systems with looseness or rubs, Chaos, Solitons \& Fractals 5 (9) (1995) 1683-1704.

http://dx.doi.org/10.1016/0960-0779(94)00171-L

[6] S. K. Sinha, Non-linear dynamic response of a rotating radial Timoshenko beam with periodic pulse loading at the free-end, International Journal of Non-Linear Mechanics 40 (1) (2005) 113149.

http://dx.doi.org/10.1016/j.ijnonlinmec.2004.05.019

[7] N. Lesaffre, J.-J. Sinou, F. Thouverez, Contact analysis of a flexible bladed-rotor, European Journal of Mechanics - A/Solids 26 (3) (2007) 541-557.

http://dx.doi.org/10.1016/j. euromechsol.2006.11.002

[8] G. Genta, On the stability of rotating blade arrays, Journal of Sound and Vibration 273 (4-5) (2004) 805-836.

http://dx.doi.org/10.1016/S0022-460x(03)00784-3

[9] E. Arnoult, Modélisation Numérique et Approche Expérimentale du Contact en Dynamique : Application au Contact Aubes/Carter de Turboréacteur, Ph.D. thesis, École Centrale de Nantes, Nantes, France (2000).

[10] P. Schmiechen, Travelling Wave Speed Coincidence, Ph.D. thesis, College of Science, Technology and Medicine, London, UK (1997).

[11] M. Legrand, B. Peseux, C. Pierre, E. Seinturier, Amélioration de la prédiction de l'interaction rotor/stator dans un moteur d'avion, Giens, France, $6^{\mathrm{e}}$ Colloque National en Calcul des Structures, 2003.

[12] T. Belytschko, W. Liu, B. Moran, Nonlinear Finite Elements for Continua and Structures, John Wiley \& Sons, 2000.

[13] A. Nayfeh, D. Mook, Nonlinear Oscillations, Willey-Interscience, New York, 1979.

[14] R. Bladh, Efficient Predictions of the Vibratory Response of Mistuned Bladed Disks by Reduced Order Modeling, Ph.D. thesis, The University of Michigan, Ann Arbor, USA (2001). http://tel .archives-ouvertes .fr/tel-00358168/fr/

[15] L. Meirovitch, Principles and Techniques of Vibrations, Prentice-Hall, Upper Saddle River, New Jersey, 1997.

[16] K.-J. Bathe, Finite Element Procedures, Prentice-Hall, Englewood Cliffs, New Jersey, 1996.

[17] D. Vola, E. Pratt, M. Raous, M. Jean, Consistent time discretization for a dynamical contact problem and complementarity techniques, Revue Européenne des Éléments Finis 7 (1-3) (1998) 149162.

[18] V. Linck, G. Bayada, L. Baillet, J. Sabil, Finite element analysis of a contact with friction between an elastic body and a thin soft layer, Journal of Tribology 127 (2005) 461-468. 
[19] N. J. Carpenter, R. L. Taylor, M. G. Katona, Lagrange constraints for transient finite element surface contact, International Journal for Numerical Methods in Engineering 32 (1) (1991) 103-128. http://dx.doi.org/10.1002/nme.1620320107

[20] P. Wriggers, Computational Contact Mechanics, Wiley, 2002.

[21] C. Pierre, A. Ferri, E. Dowell, Multi harmonic analysis of dry friction damped systems using an incremental harmonic balance method, Journal of Applied Mechanics 52 (4) (1985) 958-964. http://dx.doi.org/10.1115/1.3169175

[22] G. von Groll, D. J. Ewins, The harmonic balance method with arc-length continuation in rotor/stator contact problems, Journal of Sound and Vibration 241 (2) (2001) 223-233. http://dx.doi.org/10.1006/jsvi.2000.3298

[23] O. Poudou, C. Pierre, Hybrid Frequency-Time Domain Methods for the Analysis of Complex Structural Systems with Dry Friction Damping, in: Proceedings of the 44-th AIAA/ASME/ASCE/AHS/ASC Structures, Structural Dynamics and Material Comference, paper 2003-1411, Norfolk, Virginia, USA, 2002.

[24] S. Nacivet, C. Pierre, F. Thouverez, L. Jezequel, A dynamic Lagrangian frequency-time method for the vibration of dry-friction-damped systems, Journal of Sound and Vibration 265 (1) (2003) 201-219. http://dx.doi.org/10.1016/S0022-460X(02)01447-5

[25] T. Laursen, Computational contact and impact mechanics, Springer, Berlin, 2003. 